\title{
Cryptochrome Expression in Avian UV Cones: Revisiting the Role of Cry1 As Magnetoreceptor
}

Atticus Pinzon-Rodriguez ( $\square$ atticus.pinzon_rodriguez@biol.lu.se )

Lund University

\section{Rachel Muheim}

Lund University

\section{Research Article}

Keywords: Cryptochromes, cones, magnetoreceptor

Posted Date: November 25th, 2020

DOI: https://doi.org/10.21203/rs.3.rs-109447/v1

License: (c) (1) This work is licensed under a Creative Commons Attribution 4.0 International License. Read Full License 


\section{Abstract}

Cryptochromes (Cry) have been proposed as putative magnetoreceptors in vertebrates. Localisation of Cry 1 in the UV cones in the retinas of birds suggested that it could be the candidate magnetoreceptor. However, recent findings argue against this possibility. Cry 1 is a type II cryptochrome, a subtype of cryptochromes that may not be inherently photosensitive, and it exhibits a clear circadian expression in the retinas of birds. Here, we reassessed the localization and distribution of Cry 1 in the retina of the zebra finch. Zebra finches have a light-dependent magnetic compass based on a radical-pair mechanism, similar to migratory birds. We found that Cry1 colocalized with the UV/V opsin (SWS1) in the outer segments of UV cones, but restricted to the tip of the segments. Cry1 was expressed in all UV cones across the entire retina, with the highest densities near the fovea. Pre-exposure of birds to different wavelengths of light did not result in any difference in Cry1 expression, suggesting that Cry 1 did not undergo any detectable functional changes as result of light activation. Considering that Cry 1 is likely not involved in magnetoreception, our findings open the possibility for an involvement in different, yet undetermined functions in the avian UV/V cones.

\section{Introduction}

Cryptochromes (Cry) are flavoproteins well known for their role in the regulation of circadian activity in diverse animals (reviewed by ${ }^{1-3}$ ). They have also been proposed as the candidate magnetoreceptor proteins underlying the light-dependent magnetic compass of animals ${ }^{4}$. Magnetoreception of the lightdependent magnetic compass is suggested to be based on a radical-pair process which involves a lightinduced electron transfer between two reaction partners, and thereby the creation of an excited-state radical pair which the magnetic field can act upon ${ }^{4-8}$. Assuming that such radical-pair-based magnetoreceptors are arranged in an ordered, spherical array, with different receptors aligned at different angles to the magnetic field, the animal would be able to perceive a sensory pattern centrally symmetric

to the magnetic field lines ${ }^{4,9-11}$. Such a magnetic modulation pattern would provide information about the spatial alignment, but not the polarity, of the magnetic field lines, which is used by animals with an inclination compass, such as birds, amphibians and insects ${ }^{10,12-14}$. In birds, the eye with its semispherical shape and the highly ordered array of cells in the retina has been considered as the most likely location where magnetoreception may take place ${ }^{4,8,11}$.

Cryptochromes are the only known vertebrate photopigments that are able to form spin-correlated radical pairs upon light excitation that last long enough for a magnetic field of the strength of the Earth's magnetic field to affect the interconversion between the singlet and triplet excited states of the radical pairs $4,15,16$. Several members of the cryptochrome gene family have been found to be expressed in the retinas of both migratory and non-migratory birds ${ }^{17-24}$ : the (vertebrate) type II cryptochromes Cry 1 , with its two isoforms Cry $1 \mathrm{a}$ and Cry $1 \mathrm{~b}$, and Cry2, and the type IV cryptochrome Cry 4 and Cry4b. Until very recently, Cry1a has been considered the most promising candidate magnetoreceptor in birds. Evidence of Cry1a expression in the outer segments of SWS1-opsin (OPNSW1) expressing ultraviolet/violet (UV/V) 
cones across the retinas of domestic chickens (Gallus domesticus) and European robins (Erithacus rubecula) strongly supported an involvement of Cry $1 \mathrm{a}$ in radical-pair based magnetoreception ${ }^{25-27}$. Birds exposed to full-spectrum or monochromatic light with peak wavelengths between $373 \mathrm{~nm}$ UV and $590 \mathrm{~nm}$ yellow light prior to dissection co-expressed Cry1a and SWS1, whereas birds exposed to $645 \mathrm{~nm}$ red light or in darkness expressed only SWS1 opsin, but not Cry $1 a^{26,27}$. These findings at least partially agree with behavioural experiments showing that the light-dependent magnetic compass in birds is only operational under wavelengths between about $370 \mathrm{~nm}$ UV and $565 \mathrm{~nm}$ green light, but not under lights of longer wavelengths ${ }^{28-31}$. With the exception of the expression of Cry1a under $590 \mathrm{~nm}$ yellow light, the wavelength-dependent Cry1a expression is also compatible with the photocycle and formation of different redox states of the cryptochrome cofactor FAD (Flavin Adenine Dinucleotide). Thereby, the fully oxidized state $F A D_{O X}$ is reduced to a semi-reduced state, the neutral semiquinone radical FADHO by the absorption of light in the UV and blue spectrum (peaks at about $360 \mathrm{~nm}$ and $470 \mathrm{~nm}$ ). The neutral semiquinone radical FADHO in turn is reduced to the fully reduced state FADH- by the absorption of light from the UV to red (peaks at about $495 \mathrm{~nm}$ and $580 \mathrm{~nm}$ ), which is then re-oxidized in darkness to the fully oxidized state $\mathrm{FAD}_{\mathrm{Ox}}{ }^{32-36}$. The radical pairs can only be affected by the magnetic field in the semiquinone form, formed either during photoreduction of $F A D_{O X}$ to $F A D H O$ or during re-oxidation of $\mathrm{FADH}$ - to $\mathrm{FAD}_{\mathrm{Ox}}{ }^{34,37}$. In both Arabidopsis and Drosophila cryptochromes, light activation of FAD has been shown to result in a conformational change of the C-terminal end of the cryptochrome protein ${ }^{38,39}$. Since Niessner and colleagues observed Cry1a labelling only after exposures to UV to yellow light, but not after exposure in darkness or under red light, and their antibody was directed against the C-terminal segment of the cryptochrome, they proposed that their antibody only detected the protein after a light-triggered conformational change ${ }^{25-27}$.

Despite the convincing evidence for a role of Cry 1 in avian light-dependent magnetoreception, it has been questioned more recently whether vertebrate type II cryptochromes, which include avian Cry1, have the functional properties to act as the primary magnetoreceptors. Type II cryptochromes are widely considered to be integral parts of the negative feedback loop of the circadian clock in vertebrates by inhibiting CLOCK/BMAL1-driven ${ }^{2,3,40,41}$. Furthermore, they are believed to have a very low binding affinity to FAD and to not be intrinsically photosensitive ${ }^{42}$. Last, but not least, Cry $1 \mathrm{a}$, Cry $1 \mathrm{~b}$ and Cry 2 have been shown to exhibit circadian expression patterns in the retinas of several bird species, which does not exclude, but makes a role in avian magnetoreception less likely 20,22,23,43-46.

In view of these recent findings, we reassessed the suitability of Cry 1 as magnetoreceptor by localizing Cry 1 proteins in the retinas of zebra finches (Taeniopygia guttata, Reichenbach 1862). Zebra finches have a light-dependent magnetic compass based on a radical-pair mechanism, similar to migratory birds ${ }^{31,47-}$ ${ }^{49}$. We recently showed that in the zebra finch retina expression of Cry 1 and Cry 2 genes, unlike Cry 4 genes, exhibits a clear circadian expression profile, suggesting a role in the circadian regulation of physiological processes rather than in magnetoreception ${ }^{23}$. Here, we examined the cellular localization and distribution of Cry 1 protein across the zebra finch retina and tested whether the expression of Cry 1 
protein was wavelength dependent by examining the abundance of Cry 1 after exposure to monochromatic lights.

\section{Results}

\section{Cry1 expression in UV cones}

Evaluation of cross sections of the zebra finch retina revealed Cry 1 immunolabelled cells exclusively in the photoreceptor layer (Fig. 1A and 1B, third panel). Some non-specific signal in the inner nuclear layer did not seem to be associated to any other retinal cells, but rather intercellular matrix in that region. The strong signal visible in the photoreceptor layer in the DAPI channel is most likely due to the collapse of the pigment epithelium layer during dissection and sectioning (also noticeable from the flattened appearance of the outer segments). The retinal pigment epithelium contains lipofuscin, a known source of autofluorescence in the vertebrate retina 54,55 .

The Cry1 signal colocalized with the SWS1 immunolabelled cells (Fig. 1A and 1B, fourth and fifth panel), confirming that Cry 1 is expressed exclusively in UV cones of zebra finches, and in no other photoreceptors. Like SWS1, Cry1 was located in the outer segments of the UV cones, but it was less densely packed than SWS1 and accumulated towards the tip of the outer segment (Fig. 2). This difference in localization of Cry 1 and SWS1 signals in the outer segment is clearly visible in the bent photoreceptors of the flat-mounted, peripheral retina (Fig. 2A). It is not visible in flat mounts of the central retina due to the photoreceptors being straight (Fig. 2B).

The distribution of Cry1-positive cells was identical to the distribution of UV cones across all retinas examined (Fig. 3A). The evaluation of transects to quantify the colocalization of Cry1 and SWS1 (Fig. 3B) did not reveal any differences in expression between left or right eyes, or sex of the birds. In all evaluated retinas we found the same pattern of a peak density of the Cry1/SWS1 positive cells in the vicinity of the fovea and a concentric decline towards the periphery of the retina (Fig. 4A). The density of the co-labelled cells was slightly higher in the central, dorsal-temporal area compared to the other areas of the retina at equal distances from the fovea (Fig. 4B).

\section{Effect of monochromatic light on Cry1 expression}

We found no observable differences in the co-expression of Cry1 and SWS1 opsin, neither in the central fovea nor the periphery in any of the retinas of birds exposed to monochromatic light of $461 \mathrm{~nm}$ (blue), $521 \mathrm{~nm}$ (green) or $633 \mathrm{~nm}$ (red) prior to and during dissection (Fig 5). Cry1 was expressed in all cases irrespective of the wavelength during pre-exposure. There were no differences in Cry 1 expression between left and right eyes, or between male and female individuals.

\section{Discussion}


The aim of this study was to reassess the suitability of Cry 1 as the primary magnetoreceptor in the retina of birds. Our objectives were to identify the distribution and the cellular localization of the Cry 1 proteins in the retinas of zebra finches and to test whether the expression of Cry 1 was wavelength dependent. Our findings confirm earlier reports from migratory songbirds and chickens that Cry 1 is expressed in the outer segments of UV cones throughout the avian retina. However, we could not substantiate earlier reports of wavelength-dependent effects on Cry1 expression.

\section{Cry1 expression in outer segments of UV cones}

Our observation that Cry1 was expressed in all SWS1-labelled UV cones in the retinas of all zebra finches examined confirms earlier reports of Cry $1 \mathrm{a}$ in UV cones of European robins and $\mathrm{V}$ cones of chickens ${ }^{25-}$ 27. The occurrence of Cry 1 proteins in only the UV cones, and no other retinal tissue, in zebra finches strongly suggests that our Cry 1 antibody exclusively labelled the C-terminal of the Cry $1 \mathrm{a}$ isoform of the protein (cf. ${ }^{25-27}$. Cry $1 \mathrm{~b}$, in contrast, has been found in the retinal ganglion cells, displaced ganglion cells, and inner segments of some unspecified photoreceptors in several species of migratory songbirds and pigeons (Bolte et al. 2016; Niessner et al. 2016; see also Niessner and Winklhofer 2017).

The localisation of Cry 1 near the tip of the outer segments of the UV cones is supported by the close proximity to the UV opsins labelled with SWS1. The SWS1 antibody, like opsin antibodies in general, is known to only label the opsins once they are fixed in the membrane of the discs of the outer segment of the cones, but not while being transported from the nucleus in the inner segment to the base of the outer segment, where they are integrated in the membrane of the discs of the outer segment (reviewed by ${ }^{59}$ ). Our findings only partly agree with the study by Niessner and colleagues (2011), which reported Cry1a expression along the full length of the outer segments of $\mathrm{V}$ cones in chickens, and not only at the tip of the outer segments. This difference may originate either from species-specific results, or because the antibodies, despite being designed against a fairly similar region, are not identical. The antiserum used by Niessner and colleagues (2011) was made against a 20 aa peptide in the C-terminal region of the Cry 1 a sequence, while the antibody we used in this study was a commercial polyclonal anti-Cry 1 designed against 12 aa peptide, in the same region of the mouse Cry 1 sequence and fully homologous to their 20 aa peptide. This leaves an 8 aa peptide that is recognized by Niessner et al.'s antibody but not ours. Regardless of such differences, the localisation of the Cry1 protein far from the nucleus in the inner segment argues for a function not directly involved in the negative feedback loop of the circadian clock, one of which could be magnetoreception.

According to the original radical-pair model, the putative magnetoreceptors should ideally be fixed along at least one molecular axis and be evenly distributed across a hemisphere, like the avian retina, to allow for the comparison of reaction yields of radical pairs with different alignments relative to the magnetic field ${ }^{4,8,11}$ [note, however, that some of these requirements are not necessarily needed ${ }^{49,60-62}$ ]. These requirements are met by the localization of Cry 1 in the UV cones of the zebra finches, assuming that the Cry1 proteins are aligned roughly along the same axis in the cone outer segments. UV/V cones are in theory ideal candidate locations for cryptochrome-based magnetoreceptors, as pointed out earlier 25,63 . 
Their transparent oil droplets do not filter out UV light ${ }^{64,65}$, thus light in the UV and blue spectrum can reach the FAD in the cryptochromes, which show a high absorption of light in this wavelength range (reviewed by $2,36,37$ ). Also, UV/V cones are the least abundant photoreceptors in the avian retina (max. $10 \%$, depending on species; e.g., ${ }^{65-67}$ ), which would minimize interference of light-dependent magnetoreception with vision (but see below).

\section{Distribution of Cry1 across retina}

We found an up to nine times higher density of Cry1/SWS1 cones in the fovea of the zebra finch retina compared to the periphery, which agrees well with the general density distributions of cones in retinas of passerine birds, which usually peak in one or two foveas and decrease towards the periphery of the retina 68-71. The fovea is an important area in the visual field with a high density of cones and none or few rods 72. This area of improved visual acuity is used for various visual tasks that require high-resolution vision, like food detection or obstacle avoidance ${ }^{73}$. The higher density of Cry1-positive UV cones in the fovea of the zebra finch retina would suggest that, if they were magnetoreceptors, magnetic compass information to be perceived at a higher spatial resolution when viewed through the fovea. This may improve the detection of the magnetic field but could also pose a possible caveat in that the magnetic modulation pattern could interfere with important visual tasks. This will largely depend on how the signals from the Cry 1 and UV receptors are processed [see ${ }^{63}$, and discussion on the localisation of Cry 1 below].

\section{Effect of monochromatic light on Cry1 expression}

One of the key indications for an involvement of Cry 1 in avian magnetoreception was based on the observation that Cry 1 could only be detected in chicken retinas after exposure to UV to yellow light, but not after exposure to red light or darkness ${ }^{26,27}$. Exposure of zebra finches to $461 \mathrm{~nm}$ (blue), $521 \mathrm{~nm}$ (green) or $633 \mathrm{~nm}$ (red) light for one hour prior to dissection did not result in any differences in expression. We detected the Cry 1 protein in the retinas of zebra finches exposed to any of the light conditions, irrespective of the spectrum and the location in the retina (centre or periphery; Fig 5). This came as a surprise, since our primary antibody was designed to detect the same region (C-terminal of Cry1), and was therefore almost identical to the one used by Niessner and colleagues ${ }^{26,27}$. In both cases, the same antibody against SWS1 was used, in similar concentrations. Niessner and colleagues argued that their Cry $1 \mathrm{a}$ antibody detected the Cry 1 a protein only after it had undergone a conformational change in the C-terminal upon activation by UV to yellow light, since they detected Cry1a only after exposing the birds to UV to yellow light prior to dissection, but not after exposure to darkness or red light ${ }^{26,27}$. However, the assumption that Cry 1 undergoes a conformational change was based on evidence from plant (Arabidopsis) and type I (Drosophila) cryptochromes, which are both known to be directly light sensitive 74,75 . We are not aware of any studies showing that vertebrate type II cryptochromes undergo a conformational change in the C-terminal as a result of light activation. On the contrary, they are suggested to be vestigial flavoproteins which do not stably bind FAD and use the C-terminal for interactions with other clock proteins instead ${ }^{40-42,76}$. Assuming that the Cry 1 in the outer segments of 
the UV/V cones are located too far away from the nucleus to be directly involved in the circadian clock, the C-terminal should be detectable to antibodies under any light condition. We observed that the signal from the immunolabelled cells in some regions of our whole mounts was masked by the presence of remnants of the pigment epithelium. Despite being removed to the most extent and bleached to avoid darkening of the preparation, the pigment epithelium interfered with the fluorescent signal of the marked cells, making it almost not differentiable from the background, and therefore easy to be misinterpreted as non-labelled tissue. Own immunostainings of retinas of robins and chickens will have to show whether this may be the explanation for the discrepancies between studies, or whether differences between the antibodies or bird species are responsible.

\section{Localization of Cry1 in the avian UV/V cones suggests unique function}

Based on the cellular location of the Cry 1 proteins at the base of the outer segments of the UV cones, Cry1 could well be the thought-after candidate magnetoreceptor of the light-dependent magnetic compass. However, the high density of the Cry1-containing UV cones in the central retina is not necessarily in support of a role in magnetoreception, even though it does not exclude this possibility. The molecular and functional properties of Cry 1 also argue against its involvement in light-dependent, radicalpair-based magnetoreception. Nevertheless, the possibility remains that Cry 1 might be involved in signal transduction further downstream in the signalling cascade.

If Cry 1 is not involved in light-dependent magnetoreception in birds, why is it expressed in the outer segment of all UV cones across the entire retina of the zebra finch, but in none of the other photoreceptors or retinal cells? Together with the reports of Cry1a expression in UV cones of European robins and $V$ cones of chickens ${ }^{25-27}$ our findings suggest that Cry 1 likely has a very specific function which is unique to cones expressing the SWS1 pigment and which is not required in any of the other cone or rod photoreceptors, unless other cryptochromes are expressed in those photoreceptors instead. However, to date there is no convincing evidence that either Cry $1 \mathrm{~b}$ or Cry2 are expressed in avian photoreceptors. Cry $1 \mathrm{~b}$ has been reported in ganglion cells and a few displaced ganglion cells in retinas of pigeons (Columba livia), European robins and Northern wheatears (Oenanthe oenanthe) ${ }^{56,57}$, and possibly in the inner segment of photoreceptors, but this latter observation was only made by one of the groups ${ }^{56}$ and could not be substantiated by the other group ${ }^{57}$. Thus, all evidence points towards a very specific function of Cry 1 in only UV/V cones. Interestingly, Cry 1 has also been found to be expressed in short-wavelength sensitive SWS1 (S1) cones in representatives of some groups of mammals (Canidae, Mustelidae and Ursidae within Carnivora, Hominidae, some Cercopithecidae, and possibly Lemuridae and Callitrichidae) ${ }^{77}$. It might suggest that the expression of Cry 1 is a more widespread feature of SWS1expressing cones, common to birds and mammals, and possibly vertebrates in general.

SWS1-expressing photoreceptors are unique in that they absorb light in the UV to V spectrum ${ }^{78-80}$, which is the visible light spectrum with the highest energy and has been shown to damage the retina of vertebrates ${ }^{81}$. The vertebrate ancestral state of SWS1 opsins is suggested to be UV sensitive, but was independently replaced by $\vee$ sensitivity in various lineages (reviewed by ${ }^{78,82}$ ). These include birds which 
likely possessed an ancestral V-sensitive pigment with certain lineages secondarily regaining UV sensitivity ${ }^{82}$. Since both the UV and $V$ cones of birds contain transparent oil droplets which contain no carotenoids and do not filter short-wavelength light ${ }^{69}$, Cry 1 located in these cones could possibly be involved in UV/V-light protection. However, in mammals UV-light below $400 \mathrm{~nm}$ is often absorbed by the cornea and lens ${ }^{83}$, and there does not appear to be any relationship between Cry 1 expression and the degree of UV light below $400 \mathrm{~nm}$ reaching the SWS1 cones in different groups of mammal ${ }^{57}$.

Other possible functions of the Cry 1 proteins in the outer segments of UV/V cones in birds could be linked to the growing body of evidence that cryptochrome proteins, independent of their role as circadian clock regulators, are directly involved in various metabolic processes, like e.g., glucocorticoid signalling ${ }^{84}$, modulation of the cAMP pathway ${ }^{85}$, and DNA damage response (reviewed by ${ }^{86}$ ).

\section{Conclusions}

Based on the overall expression of Cry 1 in the UV cones of the entire retina of zebra finches, Cry 1 fulfils the requirements of the radical-pair model for a spatial expression in a hemisphere, thus could be a candidate magnetoreceptor of the light-dependent magnetic compass in birds based on this criterion alone. However, considering that Cry 1 is expressed in a clear circadian rhythm and might not be able to harvest light on its own, it is unlikely the primary magnetoreceptor initiating the radical-pair mechanism, even though the possibility remains that Cry 1 might be involved in signal transduction further downstream in the signalling cascade. Nevertheless, the unique location of Cry1 at the base of the UV cones in zebra finches suggests an exclusive function, which however remains to be determined.

\section{Materials And Methods}

\section{Experimental animals and light exposure conditions}

The zebra finches belonged to a permanent captive breeding colony at Stensoffa Field Station, near Lund (Sweden). The birds were kept indoors under full-spectrum, indoor light conditions under a constant 12 $\mathrm{h}: 12 \mathrm{~h}$ light-dark cycle for at least 7 days prior to dissection. The birds belonged to a heterogeneous group of sexually mature individuals ( $>6$ months old, 12 males and seven females) (Table S1). All retina samples were collected during the summer and autumn of 2017 and 2018 11:00 and 12:00 CET. 4 extra males were dissected for antibody validation controls and Western Blots.

The birds were terminated for tissue extraction following Swedish ethical guidelines approved by the Malmö-Lund Animal Ethics Committee (permits M 24-16, and M 108-16).

Control birds (seven males and three females) were taken directly from the holding cages for tissue collection. Birds selected for the monochromatic light experiments (five males and four females distributed among the different monochromatic light conditions - Table S1) were exposed in individual cages to one of the three wavelength spectra for $1 \mathrm{~h}$ prior to tissue collection. The dissections were done 
under the same light spectra as they were treated (full spectrum white light or monochromatic light). We used the same monochromatic light conditions as in our previous study on light-dependent magnetic compass orientation in zebra finches ${ }^{31}$ : monochromatic light with peak wavelengths at $463 \mathrm{~nm}$ blue, 521 $\mathrm{nm}$ green or $638 \mathrm{~nm}$ red produced by a LED array (OF-BLR5060RGB300, OPTOFLASH, Łódź, Poland) with a total light irradiance of $15-18 \times 10^{16}$ quanta $\mathrm{s}^{-2} \mathrm{~m}^{-2}$.

\section{Tissue collection \& whole mount preparation}

Birds were sacrificed by cervical dislocation followed by decapitation and enucleation of both eyes. Retina dissection, fixation and whole mount preparation followed the method described in ${ }^{50,51}$. Briefly, the eyes were enucleated and immediately submerged in phosphate-buffered saline (PBS) where they remained for the duration of the dissection until fixation. To access the eyecup, a circular cut was made around the ora serrata to remove the cornea and lens. The exposed eyecups were then fixed by immersion

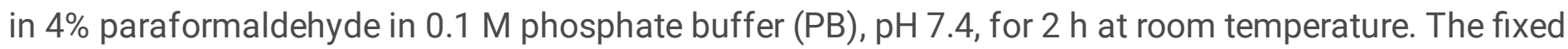
retinas were then placed in PBS, and the sclera was carefully detached and removed from the retina. To flatten the retina, six cuts were made from the periphery of the eyecup towards the centre, to alleviate the curvature. Once flat, the pecten was cut away and the pigment epithelium was mechanically removed as much as possible. Since in most samples a portion of the pigment epithelium was strongly attached to the retina, we bleached the dissected retinas in a solution of $3 \% \mathrm{H}_{2} \mathrm{O}_{2}$ in $0.1 \mathrm{M}$ PB overnight in the dark and at room temperature. The bleached retinas were then rinsed and stored in $0.1 \mathrm{M}$ PB until use. A shorter fixation (20 min) was used in a few samples (see Table S1), where the bleaching step was replaced by a dissection in PBS at $37^{\circ} \mathrm{C}$. The aim was to detach the pigment epithelium without the need for the bleaching step, exposing the tissue to a less harsh protocol.

\section{Cryosectioning}

The samples were cryosectioned following standard methods ${ }^{52}$ : fixed eyecups were cryoprotected by immersion in a $25 \%$ sucrose solution overnight at $4^{\circ} \mathrm{C}$. After this, the eyecups were changed from the sucrose solution and immersed in freezing medium (Neg-50, Richard-Allan Scientific, Thermo Fisher, Hvidovre, Denmark) at room temperature for 10 min to fully coat the tissue. The tissue was further embedded in freezing medium and frozen at $-60^{\circ} \mathrm{C}$. Semi-thin sections $(10 \mu \mathrm{m})$ were cut sequentially in a Microm HM 560 cryostat (Microm, Walldorf, Germany) along the dorsal-ventral axis. The resulting sections were thawed/mounted in chrome alum gelatine-coated microscope slides, dried overnight at room temperature, and stored at $-20^{\circ} \mathrm{C}$ until further use.

\section{Antibodies and immunostaining}

To identify which cells expressed Cry 1 , we used a rabbit polyclonal antibody against the C-terminal end of the mouse Cry1 sequence (ab3518. ABCAM, Cambridge, UK; concentration 1:100). Since the corresponding blocking Cry1 peptide from ABCAM was no longer available, we synthesized a custom peptide spanning amino acids 594 - 606 of the Mouse Cryptochrome I (QSVGPKVQRQSSN; Capra 
Science, Ängelholm, Sweden) for blocking controls and antibody validation. Specificity of the Cry1 antibody was verified by Western Blot analysis (see supplementary information). To detect UV-cones in the zebra finch retina, we used a goat polyclonal antibody against the N-terminus of the blue opsin in humans (OPN1SW; sc-14363. Santa Cruz, Dallas, TX, USA; concentration 1:250), which labels the SWS1 opsin in the UV and V cones in birds. Zebra finches have UV cones, not $V$ cones ${ }^{53}$, so we are certain that the signal of the SWS1 antibody labelled only UV cones. Primary antibodies were diluted in $1 \%$ Triton X100, 0.1 M PB solution.

Cryosections were incubated with the primary antibodies overnight at room temperature in a humid chamber. After rinsing the slides in PBS + TritonX100, the sections were incubated with the corresponding secondary fluorescent antibody [anti-rabbit Alexa 647 (1:1000) to label Cry1; anti-goat Alexa 555 (1:1000) to label SWS1 opsin; ThermoFisher] for 1 hour in darkness at room temperature. The sections were rinsed again in PBS + TritonX100, followed by PBS and then mounted with Fluoromount-G containing DAPI (SouthernBiotech, Birmingham, USA). The secondary antibody spectrum was selected to avoid autofluorescence artefacts raising from the pigment epithelium (see Results).

The whole mounted retinas were incubated with the primary antibodies overnight at room temperature under constant gentle rocking. After rinsing the retinas in $0.1 \mathrm{M} \mathrm{PB}$, the tissue was incubated with the corresponding secondary fluorescent antibody [anti-rabbit Alexa 488 (1:1000) to label Cry1; anti-goat Alexa 555 (1:1000) to label SWS1 opsin; ThermoFisher] for 2 hours in darkness at room temperature and with constant gentle rocking. The tissue was then rinsed again in $0.1 \mathrm{M} \mathrm{PB}$ and placed on gelatinized glass slides, with the photoreceptor side up, and mounted with Fluoromount-G (SouthernBiotech). Control retinas were incubated in the absence of primary antibody or with Cry 1 pre-mixed with its corresponding blocking peptide to assess specificity (Figure S2).

\section{Image acquisition and analysis}

To evaluate the presence and intracellular localization of the Cry 1 proteins, we analysed both cross sections and whole mounted retinas with a Leica SP8 DLS confocal microscope (63x/1.4 objective) with LAS-X software (Leica, Wetzlar, Germany). In addition to the immunolabelled retinal cross-sections, we acquired images of the periphery and the centre of the whole mounted retina from z-stacks spanning 9 $\mu \mathrm{m}$, starting from the base towards the tip of the outer segments of the UV cones, up to the point where the signal disappeared.

To assess the distribution of Cry1-positive cells and cones expressing SWS1 opsin throughout the retina, mounted slides were inspected on an AXIOPHOT Fluorescence Microscope (25x/0.8 and 40x/1.3 PlanNeofluar objectives; Zeiss, Oberkochen, Germany). The images were captured with a NIKON DS-fi1c CCD camera with NIS-elements software (Nikon. Tokyo, Japan). Composed images of entire retinas were reconstructed using Adobe Photoshop CS6 (San Jose, CA, USA). We established three transects with seven sampling points each to sample the photoreceptor density across the control retinas and to evaluate the degree of co-expression of Cry1 and SWS1. Each transect covered the retina from one 
periphery, passing through the central fovea all the way to the opposite periphery, so that they all intersected each other at the fovea. At each sampling point, images of each secondary antibody fluorescence were taken, and the number of positive signals was quantified using a custom particle counting software written in Matlab R2016b (The MathWorks Inc., Natick, MA, USA). The program counted positive cells on a selected region of interest of $0.1 \mathrm{~mm}^{2}$. Counts from equivalent transect points from all retinas examined were averaged and presented as a histogram, with standard deviation as error bars.

\section{Declarations}

\section{Data availability}

The data generated and analysed in the present study are included in the manuscript and its supplementary information files, or available on request from the corresponding author.

\section{Acknowlegements}

We thank Joao-Paulo Coimbra for acting as a co-advisor and teaching A.P-R. all the technical skills, like avian retina dissections and immunohistochemistry methods. This study would not have been possible without his invaluable expertise in fine tuning of the immunohistochemistry protocol and help with the interpretation of the findings. We thank Carina Rasmusson and Ola Gustafsson from the Biology Department of Lund University for invaluable technical assistance with immunohistochemical procedures and confocal imaging, respectively.

\section{Author contributions}

A.P-R. and R.M. designed the experiments. A.P-R. carried out the lab work and analysed the data. A.P-R. wrote a first draft of the manuscript. A.P-R. and R.M. wrote the final version.

\section{Competing interests}

The authors declare no competing interest.

\section{Funding}

This work was funded by Vetenskapsrådet (2011-4765 and 2015-04869 to R.M.), Crafoordska Stiftelsen (2010-1001, 2013-0737 and 2019-0839 to R.M.) and The Colombian Ministry of Science - Minciencias (formerly Colciencias: Grant 568 from Departamento Administrativo de Ciencia, Tecnología e Innovación to A.P-R.).

\section{References}


1. Cashmore, A. R. Cryptochromes: Blue Light Receptors for Plants and Animals. Science284, 760-765 (1999).

2. Chaves, I. et al. The Cryptochromes: Blue Light Photoreceptors in Plants and Animals. Annu. Rev. Plant Biol.62, 335-364 (2011).

3. Haug, M. F., Gesemann, M., Lazović, V. \& Neuhauss, S. C. F. Eumetazoan Cryptochrome Phylogeny and Evolution. Genome Biology and Evolution7, 601-619 (2015).

4. Ritz, T., Adem, S. \& Schulten, K. A Model for Photoreceptor-Based Magnetoreception in Birds. Biophysical Journal78, 707-718 (2000).

5. Schulten, K., Swenberg, C. E. \& Weller, A. A Biomagnetic Sensory Mechanism Based on Magnetic Field Modulated Coherent Electron Spin Motion. Zeitschrift für Physikalische Chemie111, 1-5 (1978).

6. Rodgers, C. T. \& Hore, P. J. Chemical magnetoreception in birds: The radical pair mechanism. Proceedings of the National Academy of Sciences106, 353-360 (2009).

7. Dodson, C. A., Hore, P. J. \& Wallace, M. I. A radical sense of direction: signalling and mechanism in cryptochrome magnetoreception. Trends in Biochemical Sciences38, 435-446 (2013).

8. Hore, P. J. \& Mouritsen, H. The Radical-Pair Mechanism of Magnetoreception. Annu. Rev. Biophys.45, 299-344 (2016).

9. Phillips, J. B., Muheim, R. \& Jorge, P. E. A behavioral perspective on the biophysics of the lightdependent magnetic compass: a link between directional and spatial perception? Journal of Experimental Biology213, 3247-3255 (2010).

10. Phillips, J. B., Jorge, P. E. \& Muheim, R. Light-dependent magnetic compass orientation in amphibians and insects: candidate receptors and candidate molecular mechanisms. J. R. Soc. Interface7, (2010).

11. Solov'yov, I. A., Mouritsen, H. \& Schulten, K. Acuity of a Cryptochrome and Vision-Based Magnetoreception System in Birds. Biophysical Journa/99, 40-49 (2010).

12. Deutschlander, M. E., Phillips, J. B. \& Borland, S. C. The case for light-dependent magnetic orientation in animals. The Journal of Experimental Biology202, 891-908 (1999).

13. Wiltschko, W. \& Wiltschko, R. Magnetic orientation and magnetoreception in birds and other animals. J Comp Physiol A191, 675-693 (2005).

14. Muheim, R. \& Liedvogel, M. The Light-Dependent Magnetic Compass. in Photobiology (ed. Björn, L. 0.) 323-334 (Springer New York, 2015). doi:10.1007/978-1-4939-1468-5_20.

15. Henbest, K. B. et al. Magnetic-field effect on the photoactivation reaction of Escherichia coli DNA photolyase. Proceedings of the National Academy of Sciences105, 14395-14399 (2008).

16. Maeda, K. et al. Chemical compass model of avian magnetoreception. Nature453, 387-390 (2008).

17. Mouritsen, H. et al. Cryptochromes and neuronal-activity markers colocalize in the retina of migratory birds during magnetic orientation. Proceedings of the National Academy of Sciences101, 1429414299 (2004). 
18. Möller, A., Sagasser, S., Wiltschko, W. \& Schierwater, B. Retinal cryptochrome in a migratory passerine bird: a possible transducer for the avian magnetic compass. Naturwissenschaften $91,585-588$ (2004).

19. Liedvogel, M. \& Mouritsen, H. Cryptochromes-a potential magnetoreceptor: what do we know and what do we want to know? J. R. Soc. Interface7, (2010).

20. Singh, D., Rani, S. \& Kumar, V. Daily Expression of Six Clock Genes in Central and Peripheral Tissues of a Night-Migratory SongBird: Evidence for Tissue-Specific Circadian Timing. Chronobiology Internationa/30, 1208-1217 (2013).

21. Fusani, L., Bertolucci, C., Frigato, E. \& Foa, A. Cryptochrome expression in the eye of migratory birds depends on their migratory status. Journal of Experimental Biology217, 918-923 (2014).

22. Günther, A. et al. Double-Cone Localization and Seasonal Expression Pattern Suggest a Role in Magnetoreception for European Robin Cryptochrome 4. Current Biology28, 211-223.e4 (2018).

23. Pinzon-Rodriguez, A., Bensch, S. \& Muheim, R. Expression patterns of cryptochrome genes in avian retina suggest involvement of Cry4 in light-dependent magnetoreception. J. R. Soc. Interface.15, 20180058 (2018).

24. Einwich, A., Dedek, K., Seth, P. K., Laubinger, S. \& Mouritsen, H. A novel isoform of cryptochrome 4 (Cry4b) is expressed in the retina of a night-migratory songbird. Scientific Reports10, 15794 (2020).

25. Niessner, C. et al. Avian Ultraviolet/Violet Cones Identified as Probable Magnetoreceptors. PLoS ONE6, e20091 (2011).

26. Niessner, C. et al. Magnetoreception: activated cryptochrome 1a concurs with magnetic orientation in birds. J. R. Soc. Interface10, 20130638 (2013).

27. Niessner, C., Denzau, S., Peichl, L., Wiltschko, W. \& Wiltschko, R. Magnetoreception in birds: I. Immunohistochemical studies concerning the cryptochrome cycle. Journal of Experimental Biology217, 4221-4224 (2014).

28. Muheim, R., Bäckman, J. \& Åkesson, S. Magnetic compass orientation in European robins is dependent on both wavelength and intensity of light. The Journal of Experimental Biology205, 3845-3856 (2002).

29. Wiltschko, R., Stapput, K., Thalau, P. \& Wiltschko, W. Directional orientation of birds by the magnetic field under different light conditions. J. R. Soc. Interface.7, (2010).

30. Wiltschko, R. et al. Orientation of migratory birds under ultraviolet light. J Comp Physiol A200, 399407 (2014).

31. Pinzon-Rodriguez, A. \& Muheim, R. Zebra finches have a light-dependent magnetic compass similar to migratory birds. J Exp Bio/220, 1202-1209 (2017).

32. Bouly, J.-P. et al. Cryptochrome Blue Light Photoreceptors Are Activated through Interconversion of Flavin Redox States. J. Biol. Chem.282, 9383-9391 (2007).

33. Liedvogel, M. et al. Chemical Magnetoreception: Bird Cryptochrome 1a Is Excited by Blue Light and Forms Long-Lived Radical-Pairs. PLoS ONE2, e1106 (2007). 
34. Kao, Y.-T. et al. Ultrafast Dynamics of Flavins in Five Redox States. J. Am. Chem. Soc.130, 1313213139 (2008).

35. Ozturk, N. et al. Comparative Photochemistry of Animal Type 1 and Type 4 Cryptochromes. Biochemistry48, 8585-8593 (2009).

36. Liu, B., Liu, H., Zhong, D. \& Lin, C. Searching for a photocycle of the cryptochrome photoreceptors. Current Opinion in Plant Biology13, 578-586 (2010).

37. Wang, J., Du, X., Pan, W., Wang, X. \& Wu, W. Photoactivation of the cryptochrome/photolyase superfamily. Journal of Photochemistry and Photobiology C: Photochemistry Reviews22, 84-102 (2015).

38. Kondoh, M. \& Terazima, M. Conformational and Intermolecular Interaction Dynamics of Photolyase/Cryptochrome Proteins Monitored by the Time-Resolved Diffusion Technique. Photochemistry and Photobiology 93, 15-25 (2017).

39. Lin, C., Top, D., Manahan, C. C., Young, M. W. \& Crane, B. R. Circadian clock activity of cryptochrome relies on tryptophan-mediated photoreduction. Proceedings of the National Academy of Sciences 201719376 (2018) doi:10.1073/pnas.1719376115.

40. Czarna, A. et al. Structures of Drosophila cryptochrome and mouse cryptochrome1 provide insight into circadian function. Cel/153, 1394-1405 (2013).

41. Ozturk, N. Phylogenetic and Functional Classification of the Photolyase/Cryptochrome Family. Photochemistry and Photobiology93, 104-111 (2017).

42. Kutta, R. J., Archipowa, N., Johannissen, L. O., Jones, A. R. \& Scrutton, N. S. Vertebrate Cryptochromes are Vestigial Flavoproteins. Sci Rep7, 44906 (2017).

43. Fu, Z., Inaba, M., Noguchi, T. \& Kato, H. Molecular cloning and circadian regulation of cryptochrome genes in Japanese quail (Coturnix coturnix japonica). J Biol Rhythms17, 14-27 (2002).

44. Haque, R., Chaurasia, S. S., Wessel, J. H. 3rd \& luvone, P. M. Dual regulation of cryptochrome 1 mRNA expression in chicken retina by light and circadian oscillators. Neuroreport13, 2247-2251 (2002).

45. Kubo, Y., Akiyama, M., Fukada, Y. \& Okano, T. Molecular cloning, mRNA expression, and immunocytochemical localization of a putative blue-light photoreceptor CRY4 in the chicken pineal gland. J Neurochem97, 1155-1165 (2006).

46. Trivedi, A. K., Malik, S., Rani, S. \& Kumar, V. Pinealectomy abolishes circadian behavior and interferes with circadian clock gene oscillations in brain and liver but not retina in a migratory songbird. Physiology \& Behavior156, 156-163 (2016).

47. Voss, J., Keary, N. \& Bischof, H.-J. The use of the geomagnetic field for short distance orientation in zebra finches. Neuroreport18, 1053-1057 (2007).

48. Keary, N. et al. Oscillating magnetic field disrupts magnetic orientation in Zebra finches, Taeniopygia guttata. Front Zoo/6, 25 (2009).

49. Muheim, R., Sjöberg, S. \& Pinzon-Rodriguez, A. Polarized light modulates light-dependent magnetic compass orientation in birds. Proc Natl Acad Sci USA113, 1654-1659 (2016). 
50. Coimbra, J. P., Marceliano, M. L. V., Andrade-da-Costa, B. L. da S. \& Yamada, E. S. The Retina of Tyrant Flycatchers: Topographic Organization of Neuronal Density and Size in the Ganglion Cell Layer of the Great Kiskadee Pitangus sulphuratus and the Rusty Margined Flycatcher Myiozetetes cayanensis (Aves: Tyrannidae). Brain Behav Evo/68, 15-25 (2006).

51. Coimbra, J. P. et al. Number and distribution of neurons in the retinal ganglion cell layer in relation to foraging behaviors of tyrant flycatchers. J. Comp. Neurol.514, 66-73 (2009).

52. Ekström, P., Garm, A., Pålsson, J., Vihtelic, T. S. \& Nilsson, D.-E. Immunohistochemical evidence for multiple photosystems in box jellyfish. Cell Tissue Res333, 115-124 (2008).

53. Hart, N. Variations in cone photoreceptor abundance and the visual ecology of birds. Journal of Comparative Physiology A: Sensory, Neural, and Behavioral Physiology187, 685-697 (2001).

54. Marmorstein, A. D., Marmorstein, L. Y., Sakaguchi, H. \& Hollyfield, J. G. Spectral Profiling of Autofluorescence Associated with Lipofuscin, Bruch's Membrane, and Sub-RPE Deposits in Normal and AMD Eyes. Invest. Ophthalmol. Vis. Sci.43, 2435-2441 (2002).

55. Double, K. L. et al. The comparative biology of neuromelanin and lipofuscin in the human brain. Cell. Mol. Life Sci.65, 1669-1682 (2008).

56. Bolte, P. et al. Localisation of the Putative Magnetoreceptive Protein Cryptochrome $1 \mathrm{~b}$ in the Retinae of Migratory Birds and Homing Pigeons. PLoS ONE11, e0147819 (2016).

57. Niessner, C. et al. Seasonally Changing Cryptochrome 1b Expression in the Retinal Ganglion Cells of a Migrating Passerine Bird. PLOS ONE11, e0150377 (2016).

58. Niessner, C. \& Winklhofer, M. Radical-pair-based magnetoreception in birds: radio-frequency experiments and the role of cryptochrome. J Comp Physiol A203, 499-507 (2017).

59. Pearring, J. N., Salinas, R. Y., Baker, S. A. \& Arshavsky, V. Y. Protein sorting, targeting and trafficking in photoreceptor cells. Progress in Retinal and Eye Research36, 24-51 (2013).

60. Hill, E. \& Ritz, T. Can disordered radical pair systems provide a basis for a magnetic compass in animals? Journal of The Royal Society Interface7, S265-S271 (2010).

61. Lau, J. C. S., Wagner-Rundell, N., Rodgers, C. T., Green, N. J. B. \& Hore, P. J. Effects of disorder and motion in a radical pair magnetoreceptor. Journal of The Royal Society Interface7, S257-S264 (2010).

62. Lau, J. C. S., Rodgers, C. T. \& Hore, P. J. Compass magnetoreception in birds arising from photoinduced radical pairs in rotationally disordered cryptochromes. J. R. Soc. Interface9, 3329-3337 (2012).

63. Bischof, H.-J., Niessner, C., Peichl, L., Wiltschko, R. \& Wiltschko, W. Avian ultraviolet/violet cones as magnetoreceptors: The problem of separating visual and magnetic information. Communicative \& Integrative Biology4, 713-716 (2011).

64. Bowmaker, J. K., Heath, L. A., Wilkie, S. E. \& Hunt, D. M. Visual pigments and oil droplets from six classes of photoreceptor in the retinas of birds. Vision Research37, 2183-2194 (1997). 
65. Hart, N. S., Partridge, J. C., Bennett, A. T. D. \& Cuthill, I. C. Visual pigments, cone oil droplets and ocular media in four species of estrildid finch. J Comp Physiol A186, 681-694 (2000).

66. Hart, N. S., Partridge, J. C. \& Cuthill, I. C. Visual pigments, oil droplets and cone photoreceptors distribution in the European Starling, Sturnus vulgaris. Journal of Experimental Biology201, 14331446 (1998).

67. Hart, N. S., Partridge, J. C., Cuthill, I. C. \& Bennett, A. T. D. Visual pigments, oil droplets, ocular media and cone photoreceptor distribution in two species of passerine bird: the blue tit (Parus caeruleus L.) and the blackbird ( Turdus merula L.). Journal of Comparative Physiology A: Sensory, Neural, and Behavioral Physiology186, 375-387 (2000).

68. Meyer, D. B. \& May, H. C. The topographical distribution of rods and cones in the adult chicken retina. Experimental Eye Research17, 347-355 (1973).

69. Hart, N. S. The Visual Ecology of Avian Photoreceptors. Progress in Retinal and Eye Research20, 675-703 (2001).

70. Coimbra, J. P., Collin, S. P. \& Hart, N. S. Variations in retinal photoreceptor topography and the organization of the rod-free zone reflect behavioral diversity in Australian passerines. Journal of Comparative Neurology523, 1073-1094 (2015).

71. Moore, B. A., Pita, D., Tyrrell, L. P. \& Fernandez-Juricic, E. Vision in avian emberizid foragers: maximizing both binocular vision and fronto-lateral visual acuity. Journal of Experimental Biology218, 1347-1358 (2015).

72. Meyer, D. B. The Avian Eye and its Adaptations. in The Visual System in Vertebrates (ed. Crescitelli, F.) vol. 7 / 5 549-611 (Springer Berlin Heidelberg, 1977).

73. Bischof, H.-J. The visual field and visually guided behavior in the zebra finch (Taeniopygia guttata). J. Comp. Physiol.163, 329-337 (1988).

74. Kondoh, M. et al. Light-Induced Conformational Changes in Full-Length Arabidopsis thaliana Cryptochrome. Journal of Molecular Biology413, 128-137 (2011).

75. Ozturk, N., Selby, C. P., Annayev, Y., Zhong, D. \& Sancar, A. Reaction mechanism of Drosophila cryptochrome. Proceedings of the National Academy of Sciences108, 516-521 (2011).

76. Merbitz-Zahradnik, T. \& Wolf, E. How is the inner circadian clock controlled by interactive clock proteins? FEBS Letters589, 1516-1529 (2015).

77. Niessner, C. et al. Cryptochrome 1 in Retinal Cone Photoreceptors Suggests a Novel Functional Role in Mammals. Sci Rep6, 21848 (2016).

78. Hunt, D. M., Carvalho, L. S., Cowing, J. A. \& Davies, W. L. Evolution and spectral tuning of visual pigments in birds and mammals. Philosophical Transactions of the Royal Society B: Biological Sciences364, 2941-2955 (2009).

79. Davies, W. I. L. et al. Vertebrate ancient opsin photopigment spectra and the avian photoperiodic response. Biology Letters8, 291-294 (2012). 
80. Hunt, D. M. \& Peichl, L. S cones: Evolution, retinal distribution, development, and spectral sensitivity. Visual NeuroscienceFirstView, 1-24 (2013).

81. Carvalho, L. S., Knott, B., Berg, M. L., Bennett, A. T. D. \& Hunt, D. M. Ultraviolet-sensitive vision in longlived birds. Proceedings of the Royal Society of London B: Biological Sciences278, 107-114 (2011).

82. Shi, Y. \& Yokoyama, S. Molecular analysis of the evolutionary significance of ultraviolet vision in vertebrates. Proceedings of the National Academy of Sciences100, 8308-8313 (2003).

83. Douglas, R. H. \& Jeffery, G. The spectral transmission of ocular media suggests ultraviolet sensitivity is widespread among mammals. Proceedings of the Royal Society of London B: Biological Sciences281, 20132995 (2014).

84. Lamia, K. A. et al. Cryptochromes mediate rhythmic repression of the glucocorticoid receptor. Nature480, 552-556 (2011).

85. Zhang, E. E. et al. Cryptochrome mediates circadian regulation of cAMP signaling and hepatic gluconeogenesis. Nature Medicine16, 1152-1156 (2010).

86. Michael, A. K., Fribourgh, J. L., Gelder, R. N. V. \& Partch, C. L. Animal Cryptochromes: Divergent Roles in Light Perception, Circadian Timekeeping and Beyond. Photochemistry and Photobiology93, 128140 (2017).

\section{Figures}


A
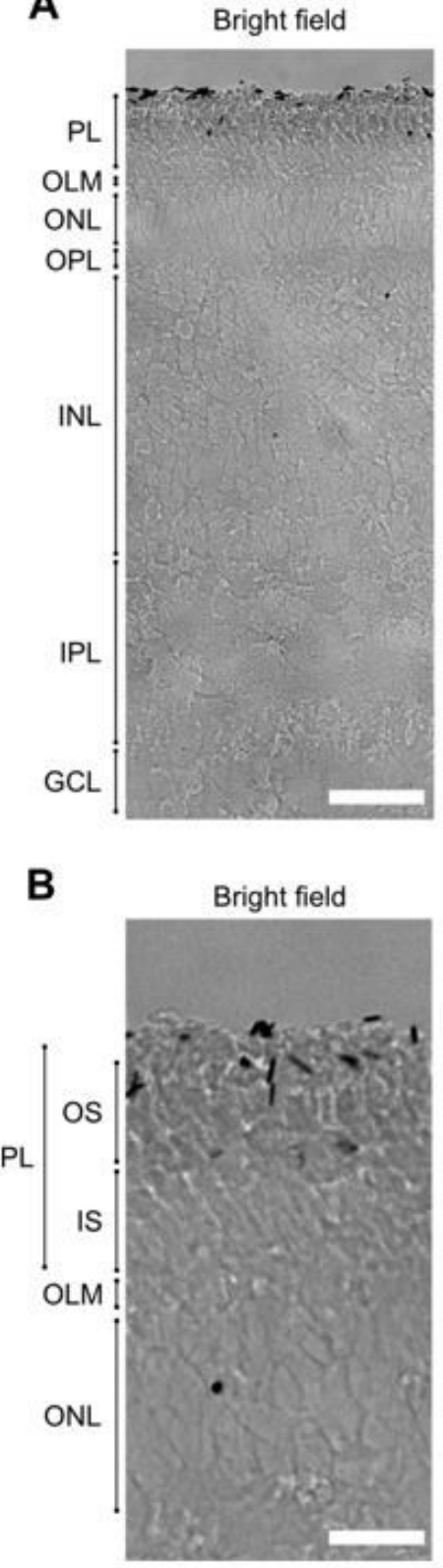

DAPI

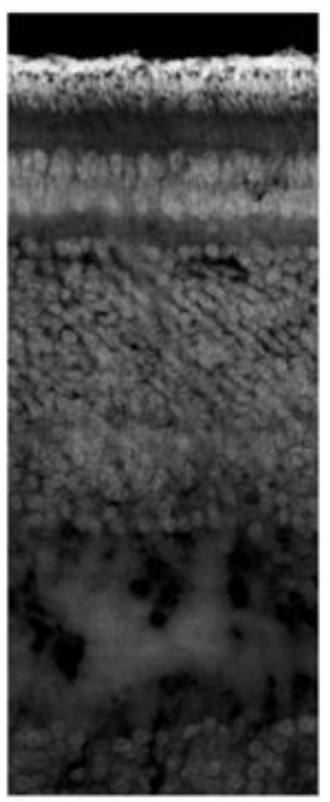

DAPI

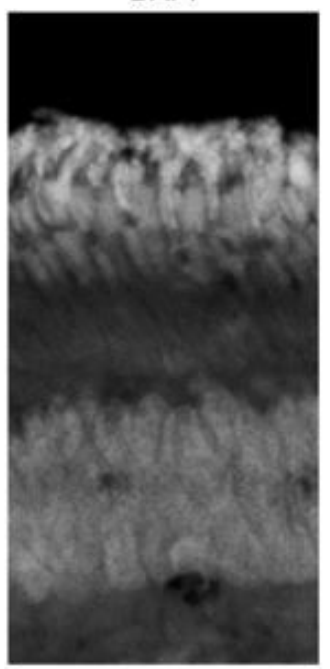

Cry1

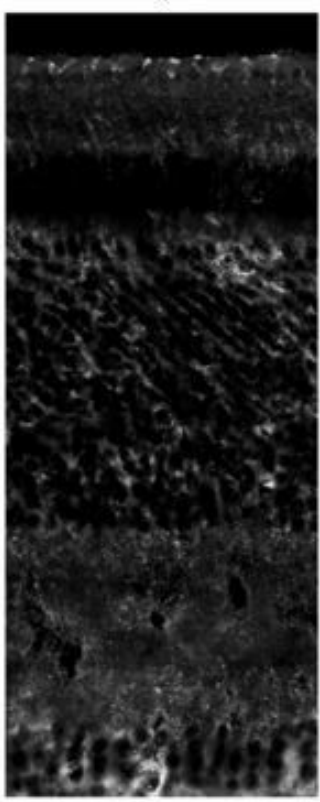

Cry1

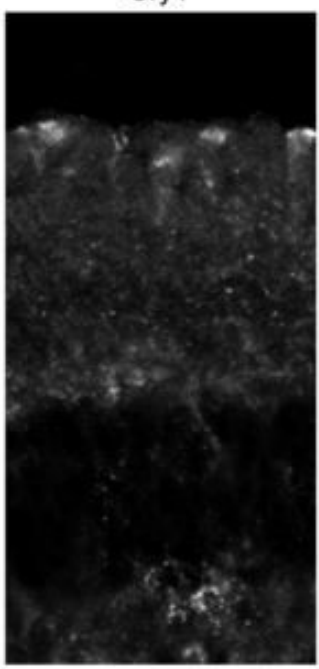

SWS1

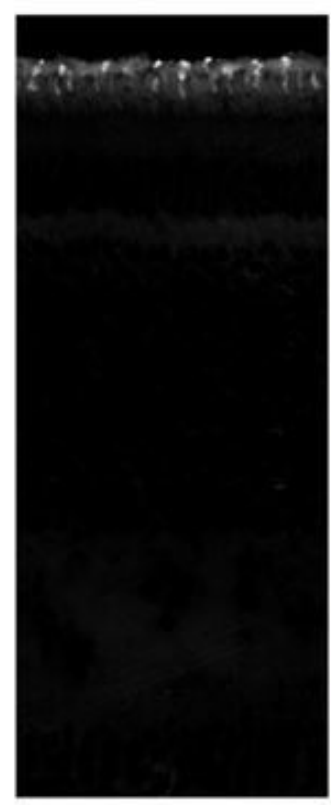

SWS1

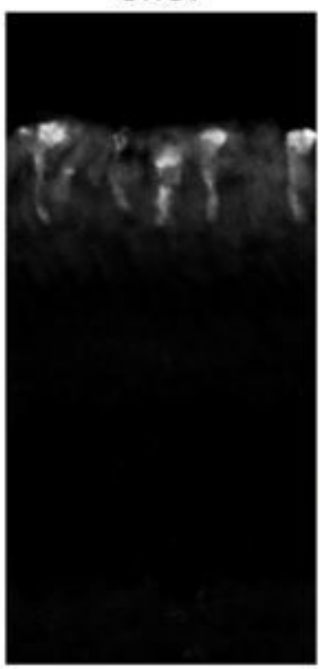

Merge

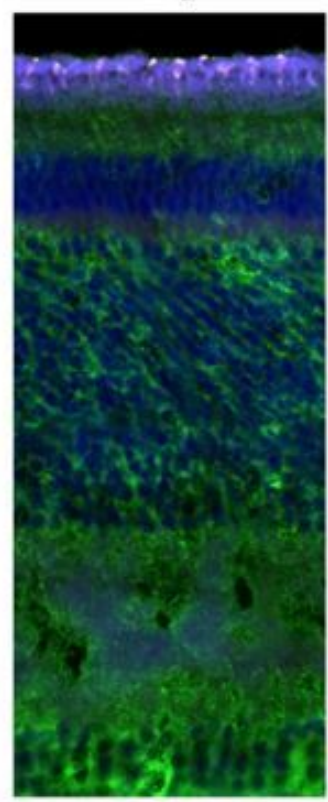

Merge

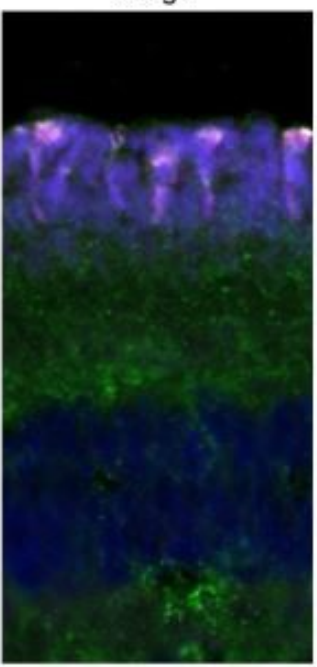

\section{Figure 1}

Cross section of the zebra finch retina. A, overview of the cross section observed with bright field microscopy (first panel) and confocal fluorescence microscopy (second to fifth panel). The fluorescence panels are merged into an artificially coloured merged image (fifth panel) in which blue corresponds to DAPI (including autofluorescence due to collapsed retinal pigment epithelium), green to Cry 1 and magenta to SWS1, with colocalization of Cry 1 and SWS1 resulting in white. B, detail of a cross section imaged as in A. PL (photoreceptor layer), OS (outer segments), IS (inner segments), OLM (outer limiting membrane), ONL (outer nuclear layer), OPL (outer plexiform layer), INL (inner nuclear layer), IPL (inner plexiform layer) and GCL (ganglion cell layer). Bar is $20 \mu \mathrm{m}$ in A and $10 \mu \mathrm{m}$ in B. 
A

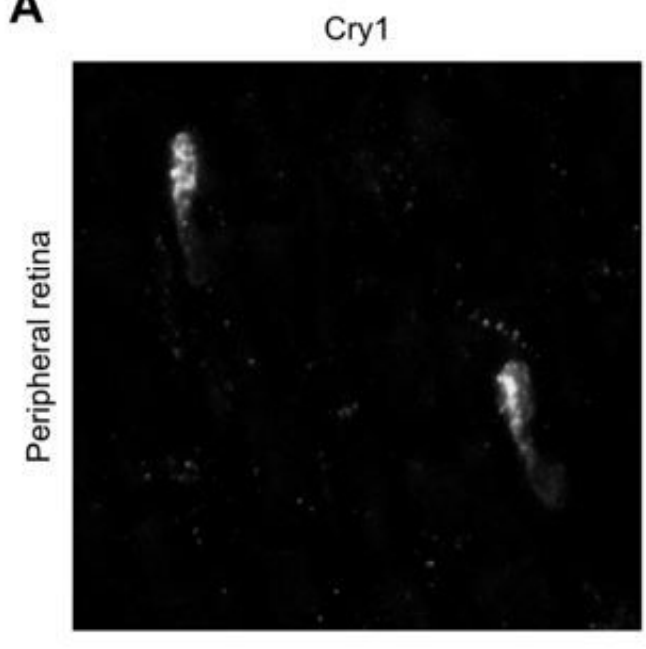

B

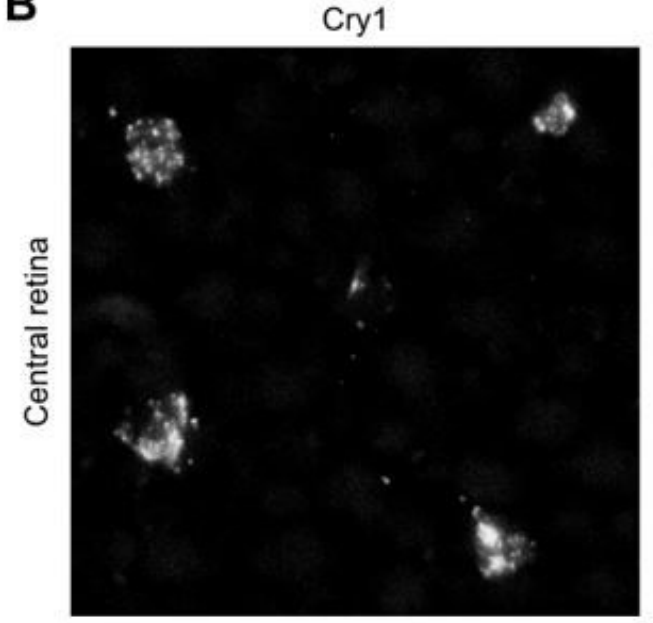

SWS1

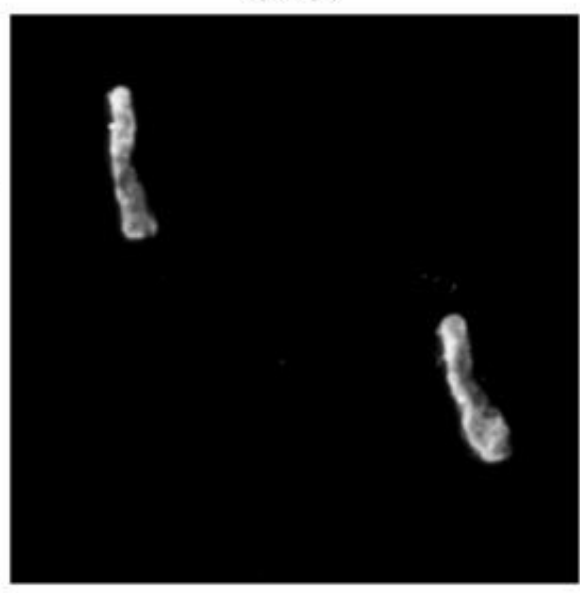

SWS1

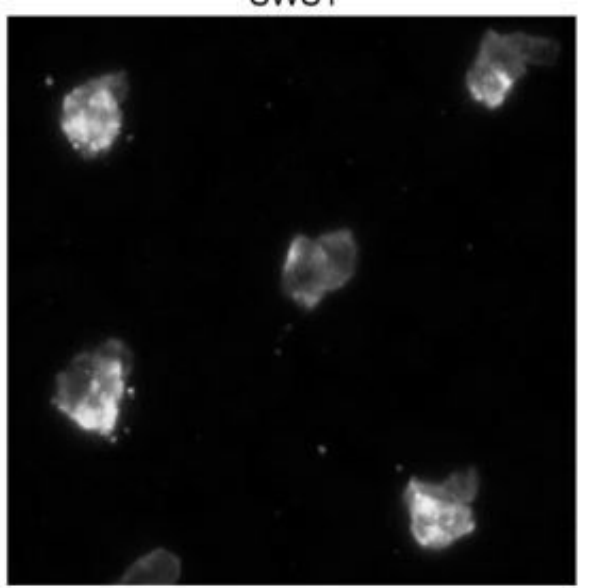

Merge

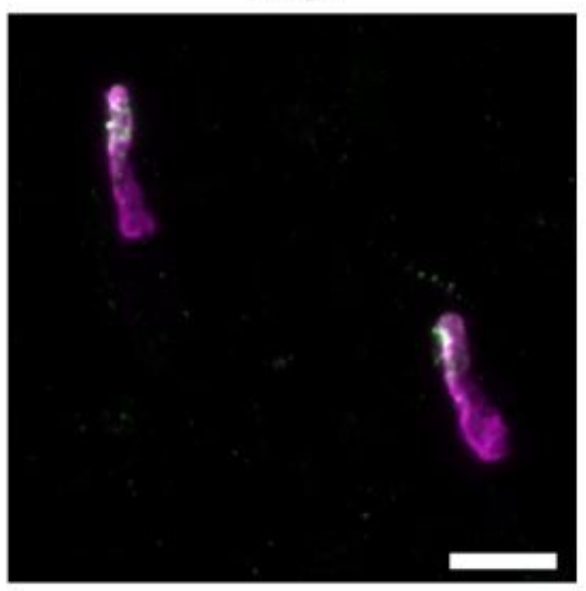

Merge

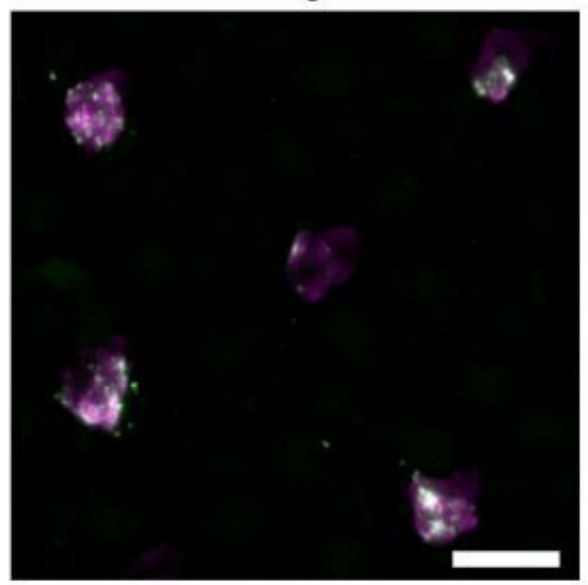

Figure 2

Outer segments of the zebra finch UV cones in a whole mount retina. A, outer segments of photoreceptors in the peripheral retina. They appear bent and elongated because of the low density of photoreceptors and the absence of pigment epithelium, which, when present, helps to keep them straight. The signal from Cry 1 appears only at the tip of the outer segment (first panel), while the UV opsin is visible across the full length of the outer segment (second panel). B, outer segments of the photoreceptors in the central retina. Here, the photoreceptors are not bent, because their high density keeps them packed and straight despite the absence of pigment epithelium. The merged images are coloured as in Figure 1. Bar $10 \mu \mathrm{m}$. 
A

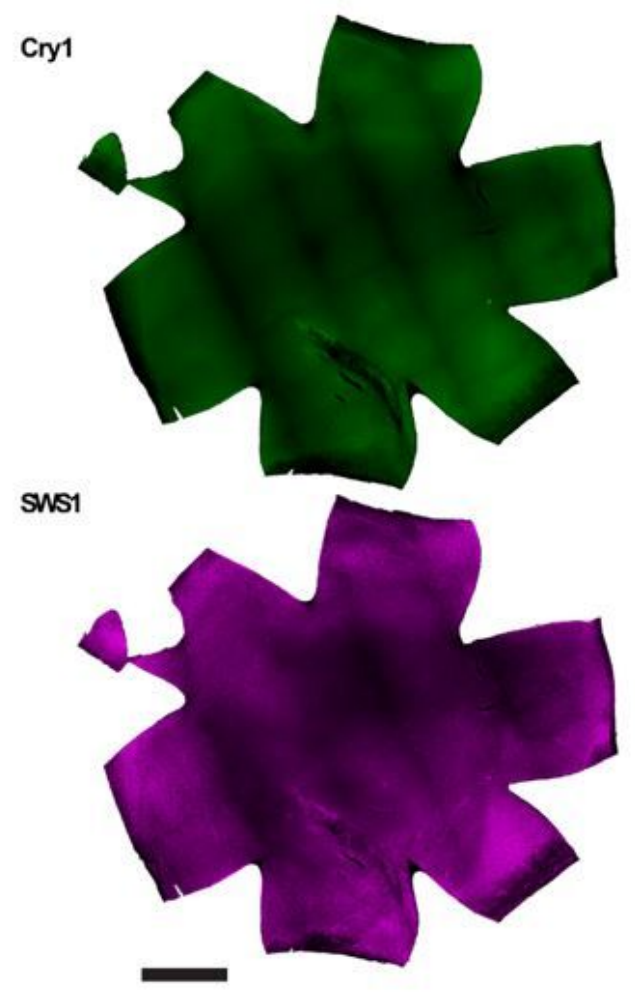

B

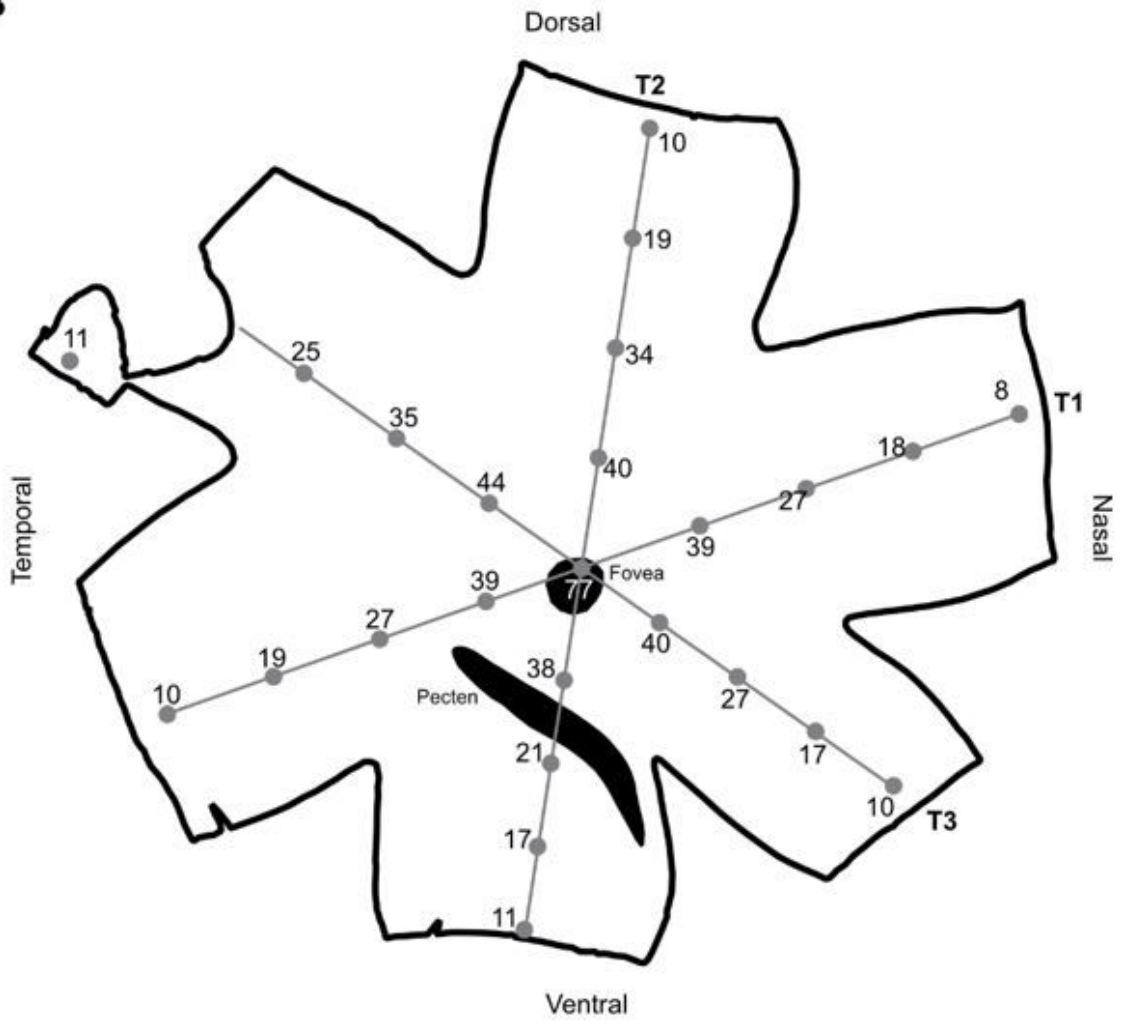

\section{Figure 3}

Example of a whole-mounted retina of a zebra finch. A, whole-mounted retina showing a positive signal for Cry1 (upper image in green) and for SWS1 opsin (lower image in purple). The image is from a left eye retina, mounted with the photoreceptors side up. The banded pattern is an artefact of the digital reconstruction of the entire image from smaller images at higher magnification. The darker regions towards the centre of the retina correspond to thicker remains of pigment epithelium. B, schematic of the entire retina shown in $A$. The grey lines and dots show the sampling transects (T1, T2 and T3) and locations where the immunopositive signals were counted. The numbers ( $x 100)$ give the counts of positive Cry1/SWS1 cells calculated for a $1 \mathrm{~mm} 2$ area at the respective locations of the retina. Bar: $2 \mathrm{~mm}$. 
A

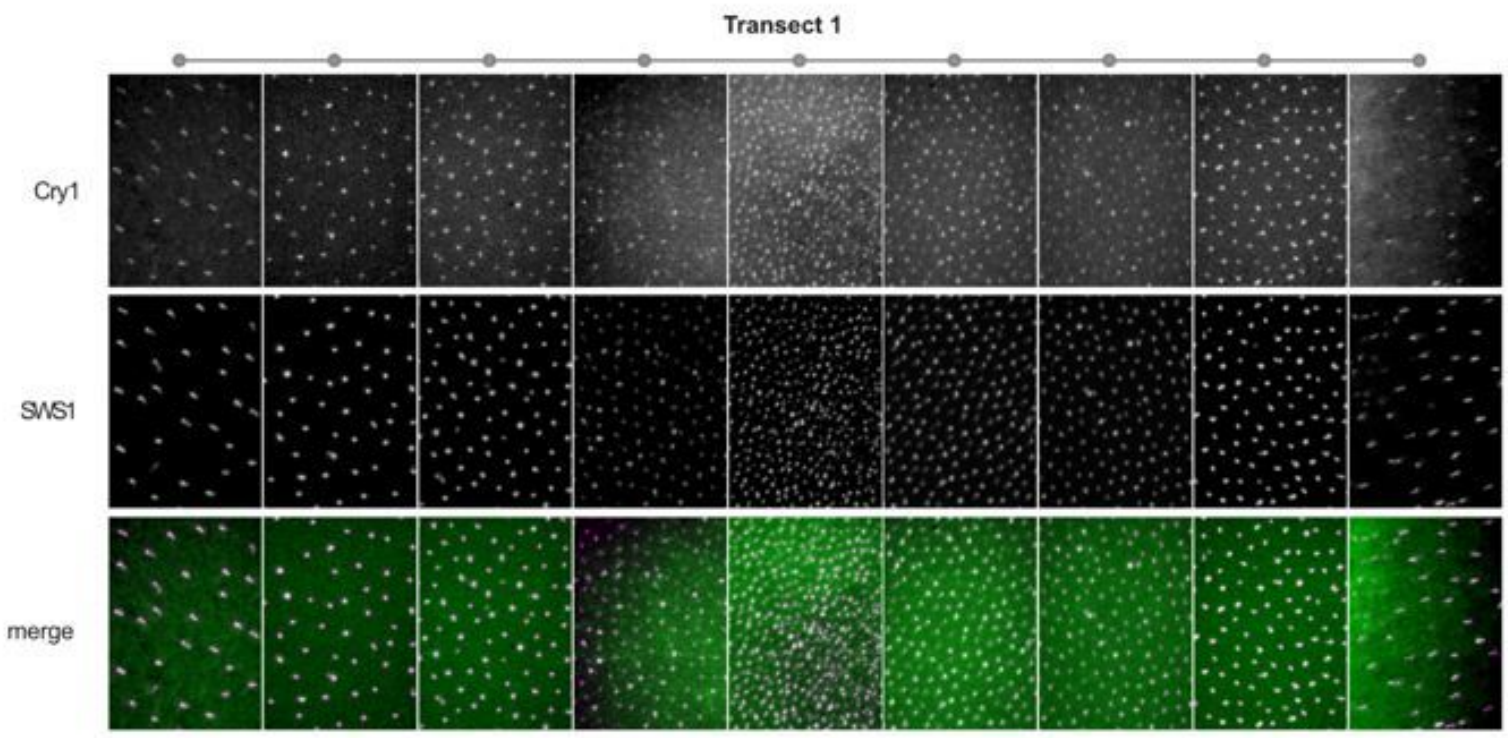

B
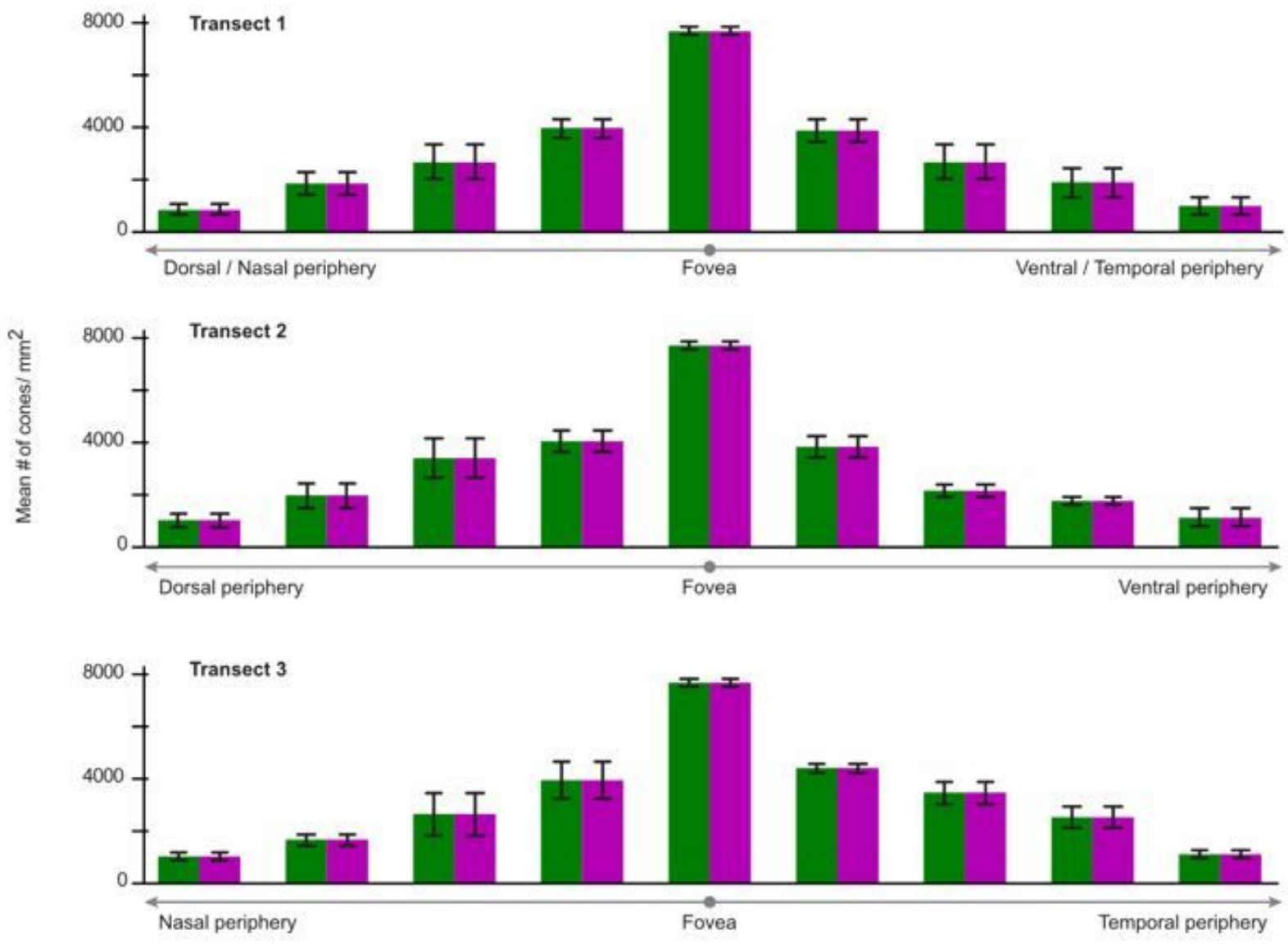

\section{Figure 4}

Quantification of the co-expression of Cry1 and SWS1 in the zebra finch retina. A, example of sampled areas across Transect 1 of a whole-mounted retina (see Fig 3B for position of sampling transects and counting locations). The upper row shows Cry1 positive cells, the middle row SWS1 positive cells, and the bottom row the merged images (Cry1 in green, SWS1 in purple and colocalization is seen as white). B, mean number ( \pm standard deviations) of immunopositive cells per $\mathrm{mm} 2$ for each sampling location 
along the three transects. The counts are based on six retinas from four individual birds (see Table S1). Bar: $30 \mu \mathrm{m}$.

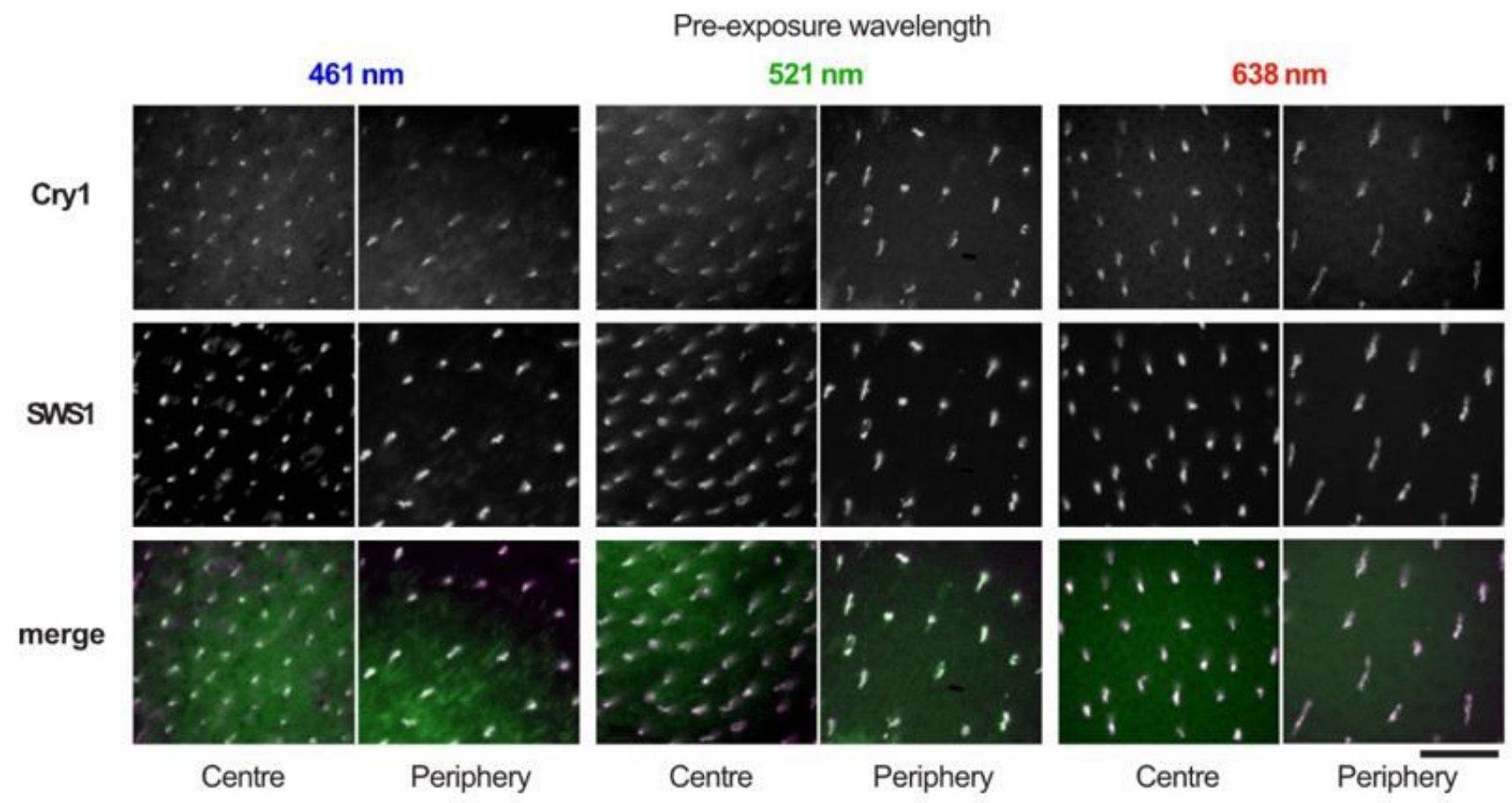

\section{Figure 5}

Co-expression of Cry 1 and SWS1 in retinas of zebra finches exposed to monochromatic illumination. The upper row shows Cry1-positive cones, the middle row SWS1-positive cells, and the bottom row shows the merged images (Cry1 in green, SWS1 in purple and colocalization is seen as white). The panels illustrate examples from birds exposed to $461 \mathrm{~nm}$ blue light (left), $521 \mathrm{~nm}$ green light (centre) and $638 \mathrm{~nm}$ red light (right). For each wavelength spectrum, an example from the central retina and one from the peripheral retina is shown. Bar: $30 \mu \mathrm{m}$.

\section{Supplementary Files}

This is a list of supplementary files associated with this preprint. Click to download.

- ManuscriptCry1inUVconesSciRepformatSI.pdf 